\section{(6) OPEN ACCESS}

\title{
Optimising the cost and delivery of HIV counselling and testing services in Kenya and Swaziland
}

\author{
Carol Dayo Obure, ${ }^{1}$ Anna Vassall, ${ }^{1}$ Christine Michaels, ${ }^{1}$ Fern Terris-Prestholt, ${ }^{1}$ \\ Susannah Mayhew, ${ }^{1}$ Lucy Stackpool-Moore, ${ }^{2}$ Charlotte Warren, ${ }^{3}$ The Integra research \\ team, Charlotte Watts ${ }^{1}$
}

\begin{abstract}
- An additional appendix is published online only. To view this file please visit the journal online (http://dx.doi.org/ 10.1136/sextrans-2012-050544).

${ }^{1}$ Social and Mathematical Epidemiology Group (SAME), Department of Global Health and Development, London School of Hygiene and Tropical Medicine, London, UK ${ }^{2}$ International Planned Parenthood Federation (IPPF), London, UK

${ }^{3}$ Population Council, Nairobi, Kenya
\end{abstract}

\section{Correspondence to}

Anna Vassall, Department of Global Health and Development, London School of Hygiene and Tropical Medicine, 15-17 Tavistock Place, London, WC1H 9SH, UK;

anna.vassall@Ishtm.ac.uk

Accepted 30 June 2012

Published Online First 3 August 2012

\section{ABSTRACT}

Background Approaches to HIV counselling and testing (HCT) within low-resource high HIV prevalence settings have shifted over the years from primarily client-initiated approaches to provider initiated. As part of an ongoing programme science research agenda, we examine the relative costs of provider-initiated testing and counselling (PITC) services compared with voluntary counselling and testing (VCT) services in the same health facilities in two low-resource settings: Kenya and Swaziland.

Methods Annual financial and economic costs and output measures were collected retrospectively from 28 health facilities. Total annual costs and average costs per client counselled and tested (C\&T), and HIV-positive clients identified, were estimated.

Results VCT remains the predominant mode of HCT service delivery across both countries. However, unit cost per client C\&T and per person testing HIV positive is lower for PITC than VCT across all facility types in Kenya, but the picture is mixed in Swaziland. Average cost per client C\&T ranged from US\$4.81 to US\$6.11 in Kenya, US\$6.92 to US\$13.51 in Swaziland for PITC, and from US $\$ 5.05$ to US\$16.05 and US\$8.68 to US\$19.32 for VCT in Kenya and Swaziland, respectively.

Conclusions In the context of significant policy interest in optimising scarce HIV resources, this study demonstrates that there may be potential for substantial gains in efficiency in the provision of HCT services in both Kenya and Swaziland. However, considerations of how to deliver services efficiently need to be informed by local contextual factors, such as prevalence, service demand and availability of human resources.

\section{INTRODUCTION}

Despite significant progress, sub-Saharan Africa (SSA) continues to have the highest global burden of HIV infection. ${ }^{1}$ With the roll out of antiretroviral treatment, and recognition that early HIV treatment may also impact on future HIV transmission, HIV counselling and testing (HCT) is an important cornerstone of HIV programming. ${ }^{2-6}$ The early investment in stand-alone voluntary counselling and testing (VCT) was supported by research findings suggesting that the provision of VCT is cost-effective. ${ }^{7}$ However, using the VCT model, the uptake of HCT in many high HIV prevalence countries in SSA remained low, with the poor uptake being due to both demand factors (such as fear of stigma associated with accessing stand-alone
HIV services); and supply factors (including the limited availability of testing centres in many settings). ${ }^{5} 89$ Many SSA countries are, therefore, currently exploring and scaling up alternative approaches to HCT to encourage uptake of HIV testing to population groups with limited access to existing services.

In addition, amidst the current economic crisis, there is a renewed interest in achieving 'value for money', with policy makers focusing on the most efficient way of delivering key HIV services without compromising quality. For countries seeking to expand HCT coverage, integrating HCT services into existing services, using a providerinitiated testing and counselling (PITC) approach, offers the potential to reduce HIV-related service costs. This study seeks to add to the evidence on the efficiency of delivering HCT services in lowresource settings by comparing the economic costs of delivering HCT services through PITC and VCT at 28 health facilities in Kenya and Swaziland, as part of a larger project, Integra Initiative.

Drawing on key programme science principles, ${ }^{10}$ this joint project between researchers and implementers supports the planning and delivery of integrated HIV and sexual reproductive health (SRH) services; from programme design to assessing the impact of integration key outcomes and service delivery goals, including the assessment of the efficiency of SRH and HIV services (http:// www.integrainitiative.org)

\section{STUDY SETTING}

Kenya has a generalised HIV epidemic with a prevalence rate of $7.1 \%$, according to the last Kenya AIDS Indicator Survey (2007). ${ }^{11}$ On the other hand, with an estimated adult HIV prevalence of $26 \%$, Swaziland has the world's most severe generalised HIV epidemic. ${ }^{12}$ HCT is central to both Kenya and Swaziland's national response to HIV. However, despite the increasing availability of VCT centres, knowledge of HIV status remains low. ${ }^{13} 14$ In a bid to encourage HCT uptake to population groups with limited access to existing services, the Ministries of Health of both Kenya and Swaziland mandated PITC throughout the health sector in 2008. The Integra Initiative further strengthened this effort by providing staff training, equipment, supplies and supportive supervision to improve delivery of HTC services within SRH services. 


\section{VCT and PITC Provision}

VCT refers to the client-initiated counselling and testing for HIV through VCT centres, while PITC involves the incorporation of HCT into routine healthcare, including general primary care, maternal and child healthcare, care for sexually transmitted infections and inpatient services. Both VCT and PITC services are offered in Kenya and Swaziland, and follow similar testing procedures in both countries.

There are, however, several differences between the PITC and VCT models, which have associated resource implications. While PITC services are routinely offered to all clients attending services, regardless of their reason for accessing services, VCT is dependent upon clients seeking testing. Within VCT, counselling and testing is provided by a lay VCT counsellor or a nurse. For PITC, pre- and post-test counselling is provided by a nurse, and testing is conducted either by the same nurse or provided by a laboratory technologist or a lay counsellor. VCT generally involves one-on-one or couples counselling, while for PITC pretest counselling may be provided to groups. PITC may also involve much less post-test counselling than VCT, and thus, requires a shorter length of staff time for each visit.

\section{Study sites}

The study was conducted in a total of 41 health facilities in Kenya and Swaziland. Of the 41 sites, only 28 provided both PITC and VCT services within the same facility. Facilities were purposively selected to represent different locations (urban and rural), different ownership types (government and private notfor-profit) and different types of facilities (hospitals, district hospitals, sub-district hospitals, health centres, general clinics and International Planned Parenthood Federation affiliated SRH clinics). An overview of the study sites, and their size, in terms of staffing numbers and overall outpatient visits, is provided in the online appendix. Ethics approval for the larger Integra Initiative was obtained from the Kenya Medical Research Institute National Ethical Review Committee, and the Swaziland Scientific Review Board.

\section{METHODOLOGICAL APPROACH}

Cost and output data for both services were collected retrospectively at the facility level for the 2008-2009 fiscal year from financial records and routine monitoring data. A combination of standard step-down and micro-costing methods was used to estimate the financial and economic costs of providing HCT services from a health provider perspective. (Financial costs represent actual expenditures on goods and services purchased, while economic costs include the estimated value of all resources, including donated or subsidised goods and services). Costs were classified as capital or recurrent costs.

Capital costs included costs of space, furniture and equipment and staff training. Equipment and furniture replacement value was obtained from the Ministry of Health for the public facilities, and retail sellers for the private health facilities, and costs were annuitised using a discount rate of $3 \%{ }^{15}$

Recurrent costs included staff costs, building maintenance, communications and stationery, diagnostics and supply. Where staff were shared across different activities, time usage was measured by a combination of observation, interviews with staff and their managers, and was confirmed by examining records on clients seen. Other items were measured through a combination of observation of resource use and supplies, and expenditure records.

Unit costs were obtained by dividing total costs by the relevant output indicators. Local currencies were converted to US dollars (\$) using an exchange rate of 78.79 Kenyan shillings per US dollar (\$), and 7.85 Swaziland emalangeni per US dollar. ${ }^{16}$ All costs are presented in US dollar rates prevailing in 2009. Data analysis was conducted using Microsoft Excel 2007 and Stata (V.11.0: Stata Corporation).

\section{RESULTS}

The provision of HCT services varies considerably across facilities in both countries. Table 1 presents the summary of service outputs by facility type in Kenya and Swaziland. In Kenya, VCT was found to be the predominant mode of delivery of HCT, particularly at the district hospital and private SRH clinics. In Swaziland, the picture was mixed. The public facilities recorded higher proportions of VCT clients, whereas, the private SRH clinics reported more people accessing PITC services.

The proportion of clients seeking counselling, who then received a test, was almost $100 \%$ for both VCT and PICT services in both countries; except for PITC at the private SRH clinics. Only

Table 1 Average (mean) outputs for each facility type in Kenya and Swaziland

\begin{tabular}{|c|c|c|c|c|c|}
\hline Kenya $(\mathrm{N}=20)$ & Provincial hospital $(n=1)$ & District hospital $(n=5)$ & Sub-District hospital $(n=5)$ & Health centre $(n=4)$ & $\begin{array}{l}\text { Private SRH } \\
\text { clinic }(n=5)\end{array}$ \\
\hline \multicolumn{6}{|l|}{ PITC } \\
\hline Clients counselled only & 3094 & 942 & 419 & 779 & 562 \\
\hline Clients HIV positive (\% of total C\&T) & $488(16)$ & $145(15)$ & $42(10)$ & $105(14)$ & Not available \\
\hline \multicolumn{6}{|l|}{ VCT } \\
\hline Clients counselled only & 3042 & 2416 & 664 & 519 & 2374 \\
\hline Swaziland ( $N=8$ ) & Hospital $(n=1)$ & Health centre & Public heal & unit $(n=1)$ & $\begin{array}{l}\text { Private SRH } \\
\text { clinic }(n=2)\end{array}$ \\
\hline \multicolumn{6}{|l|}{ PITC } \\
\hline Clients counselled only & 1657 & 303 & 1976 & & 2134 \\
\hline Clients C\&T (\% of total counselled) & $1657(100)$ & $303(100)$ & $1976(100)$ & & $936(44)$ \\
\hline Clients HIV positive (\% of total C\&T) & $1200(43)$ & $523(41)$ & $887(65)$ & & $88(15)$ \\
\hline
\end{tabular}


$44 \%-48 \%$ of clients counselled through PITC were also tested in the SRH clinic. The proportion testing positive was found to be particularly high for VCT at the provincial hospital in Kenya, and for all HCT in Swaziland throughout the public facilities.

The total annual facility average costs of delivering HCT services and cost profiles across all facility types are summarised in table 2. In Kenya, the average total annual costs ranged between US\$1863 in the SRH clinics and US\$16723 in the provincial hospital for PITC; and US\$2768 in the sub-district hospitals and US\$48836 in the provincial hospital for VCT services. The largest components of costs for PITC across facility types were personnel costs $(35 \%-49 \%)$ and recurrent supplies costs (which include diagnostics and supplies) (44\%-62\%). Similarly for VCT, the main cost component across facility types was personnel $(41 \%-64 \%)$ and recurrent supplies $(32 \%-53 \%)$. For both PITC and VCT services, capital and other recurrent costs were low.

In Swaziland, the average total annual costs ranged between US\$3233 in the health centres and US\$22 362 in the hospital for PITC. For VCT, the average total annual costs ranged from US \$9767 in the SRH clinics to US\$54 414 in the hospital. As with Kenya, the major cost components for HTC services across all facility types were recurrent supplies costs $(39 \%-87 \%$ for PITC and $28 \%-70 \%$ for VCT) and personnel costs $(11 \%-60 \%$ for PITC and $13 \%-70 \%$ for VCT).

Table 3 presents a breakdown of average cost per PITC and VCT client counselled and tested (C\&T), and the average cost per HIV-positive diagnosis. The average cost per client C\&T (including diagnostics and supplies) through PITC ranged from US\$4.81 in the health centres to US\$6.11 in the SRH clinics in Kenya, and from US\$6.92 in the PHU to US\$13.51 in the SRH clinic in Swaziland. Average costs per client C\&T through VCT ranged from US\$5.05 in the health centres to US\$16.05 in the provincial hospital, and from US\$8.68 in the PHU to US\$19.32 in the hospital in Kenya and Swaziland, respectively.

Figure 1 shows the comparison of unit costs by HCT service and facility type in both Kenya and Swaziland. Costs per client C\&T through VCT are generally higher than cost per PITC client C\&T across the different facility types in Kenya. In contrast in Swaziland, the unit costs per PITC were higher than unit costs per VCT client C\&T in three of the health centres and the SRH clinics.

The cost per HIV-positive client identified ranges from US $\$ 34.27$ to US\$140.55 in Kenya, and US\$13.28 and US\$126.88 in Swaziland (table 3). In Kenya, the cost per client diagnosed as HIV positive through PITC and VCT was lowest at the provincial hospital. In Swaziland, the cost per client diagnosed as HIV positive through both PITC and VCT approaches was lowest in the PHU at US\$20.26 and US\$13.28, respectively.

Aside from capital costs in health centres in Swaziland, most of the variation in average costs per client $C \& \mathrm{~T}$ is driven by costs of human resources. We found little variation in salaries costs (including cadre of staff used). To explain the variation in personnel costs between facilities and different approaches to HCT, staff workload was measured by the number of clients C\&T per staff full-time equivalency. A correlation analysis across both countries between the unit salaries cost per HCT visit, and this measure of staff workload shows a negative coefficient (0.39631) with a $p$ value of $(0.0025)$.

\section{DISCUSSION}

Our findings suggest that in Kenya and Swaziland, PITC services compare favourably with VCT in terms of cost per client C\&T

Table 2 Annual economic cost of delivering VCT and PITC services for each facility type (US\$ rates in 2009)

\begin{tabular}{|c|c|c|c|c|c|c|}
\hline Type of cost (Average, US\$) & $\begin{array}{l}\text { Provincial } \\
\text { hospital }(n=1)\end{array}$ & $\begin{array}{l}\text { District } \\
\text { hospital }(n=5)\end{array}$ & $\begin{array}{l}\text { Sub-district } \\
\text { hospital }(n=5)\end{array}$ & $\begin{array}{l}\text { Health centre } \\
(n=4)\end{array}$ & $\begin{array}{l}\text { Private SRH } \\
\text { clinic }(n=5)\end{array}$ & Average \\
\hline \multicolumn{7}{|l|}{ Kenya } \\
\hline \multicolumn{7}{|l|}{ PITC Service } \\
\hline Capital cost (\% of total cost) & $126(1)$ & $91(2)$ & $54(2)$ & $118(3)$ & $152(8)$ & $104(3)$ \\
\hline Personnel cost (\% of total cost) & $8066(48)$ & $2533(49)$ & $568(35)$ & $1096(35)$ & $793(43)$ & $1624(44)$ \\
\hline Recurrent supplies (\% of total cost) & 7911 (47) & 2416 (47) & $1106(62)$ & $2081(61)$ & $821(44)$ & $1898(51)$ \\
\hline Annual economic cost & 48836 & 16341 & 5699 & 2768 & 13086 & 11969 \\
\hline Capital cost (\% of total cost) & $276(1)$ & $125(1)$ & $86(2)$ & $87(3)$ & 1159 (9) & $374(3)$ \\
\hline Personnel cost (\% of total cost) & $29093(59)$ & $10398(59)$ & $3285(64)$ & $1200(42)$ & $5435(41)$ & $6474(54)$ \\
\hline Recurrent supplies (\% of total cost) & $16675(34)$ & $6590(37)$ & $1633(32)$ & $1511(53)$ & $5232(40)$ & $4500(38)$ \\
\hline Other recurrent (\% of total cost) & $2791(6)$ & $540(3)$ & $78.53(2)$ & $58(2)$ & $1260(10)$ & $621(5)$ \\
\hline Capital cost (\% of total cost) & $57(0.3)$ & $504(16)$ & $93(1)$ & $318(2)$ & & $351(3)$ \\
\hline Personnel cost (\% of total cost) & $13073(60)$ & $1137(35)$ & $1451(11)$ & $3658(21)$ & & $3299(32)$ \\
\hline Recurrent supplies (\% of total cost) & $8582(39)$ & $1539(48)$ & $12055(87)$ & 8411 (48) & & $5452(52)$ \\
\hline Other recurrent (\% of total cost) & $181(0.8)$ & $52(2)$ & $74(1)$ & $4992(29)$ & & $1306(13)$ \\
\hline \multicolumn{7}{|l|}{ VCT Service } \\
\hline Annual economic cost & 54414 & 12003 & 11777 & 9767 & & 16716 \\
\hline Capital cost (\% of total cost) & $242(0.4)$ & $113(1)$ & $598(5)$ & $89(1)$ & & $184(1)$ \\
\hline Personnel cost (\% of total cost) & $38032(70)$ & 4609 (38) & $2674(23)$ & $1282(13)$ & & $7713(46)$ \\
\hline Recurrent supplies (\% of total cost) & $15366(28)$ & $6585(55)$ & $8314(70)$ & $4713(48)$ & & $7431(44)$ \\
\hline Other recurrent (\% of total cost) & $773(1)$ & $696(6)$ & $191(2)$ & 3681 (38) & & $1389(8)$ \\
\hline
\end{tabular}


Table 3 Average unit cost per person counselled and tested, breakdown of unit cost by input type and cost per person testing positive (US\$ rates in 2009)

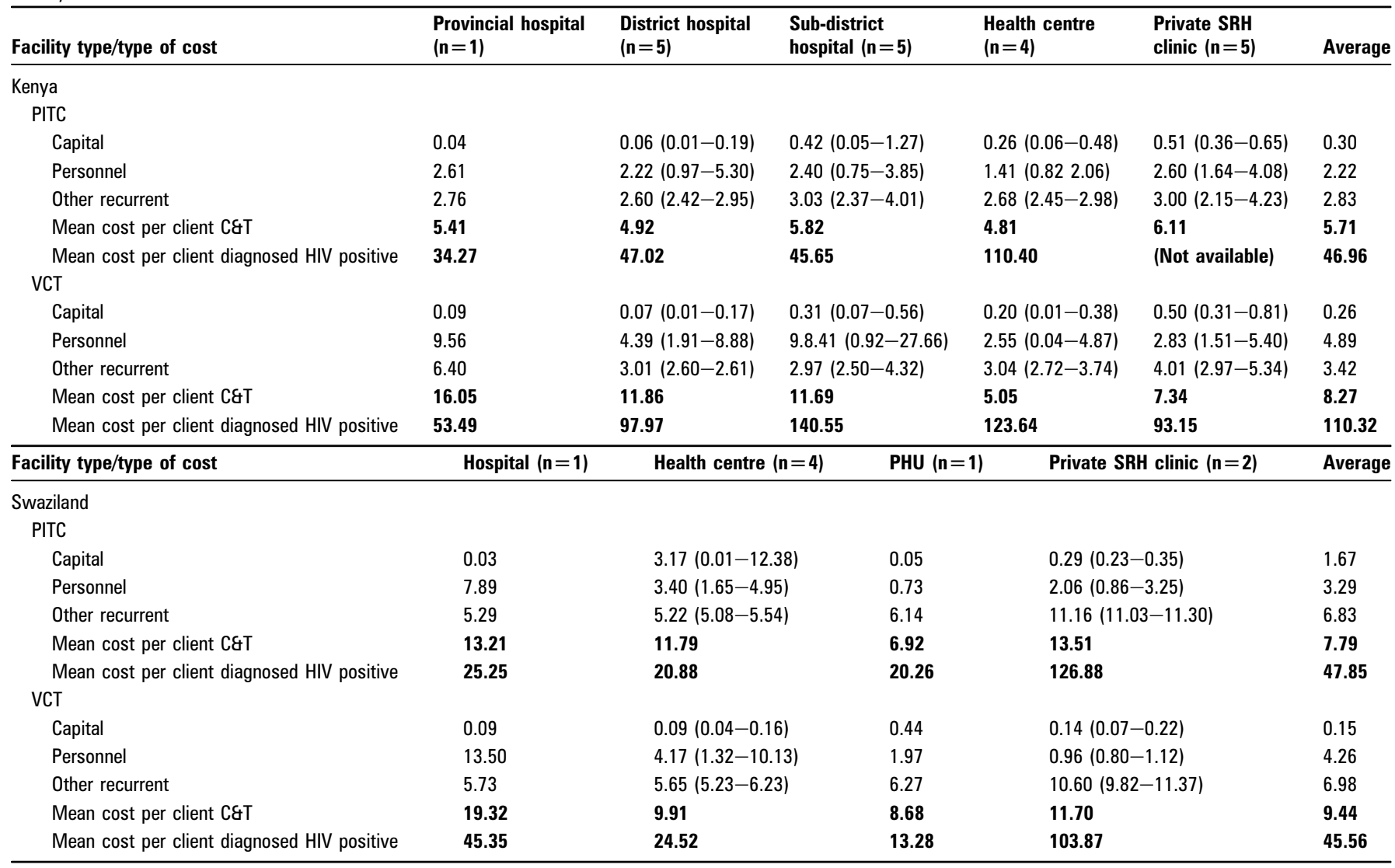

and cost per client diagnosed as HIV positive. In Kenya, we find that VCT at hospitals tends to identify proportionally more clients who are diagnosed as HIV positive than PITC. However, the picture is slightly different in Swaziland, where all levels of publicly owned services find high proportions of HIV positives among those tested $(34 \%-65 \%)$. The exceptions to this are the private SRH clinics. The private clinics also have a much lower proportion of those receiving counselling than going onto being tested; possibly revealing the more voluntary nature of their provider initiative testing. Unfortunately, we were not able to

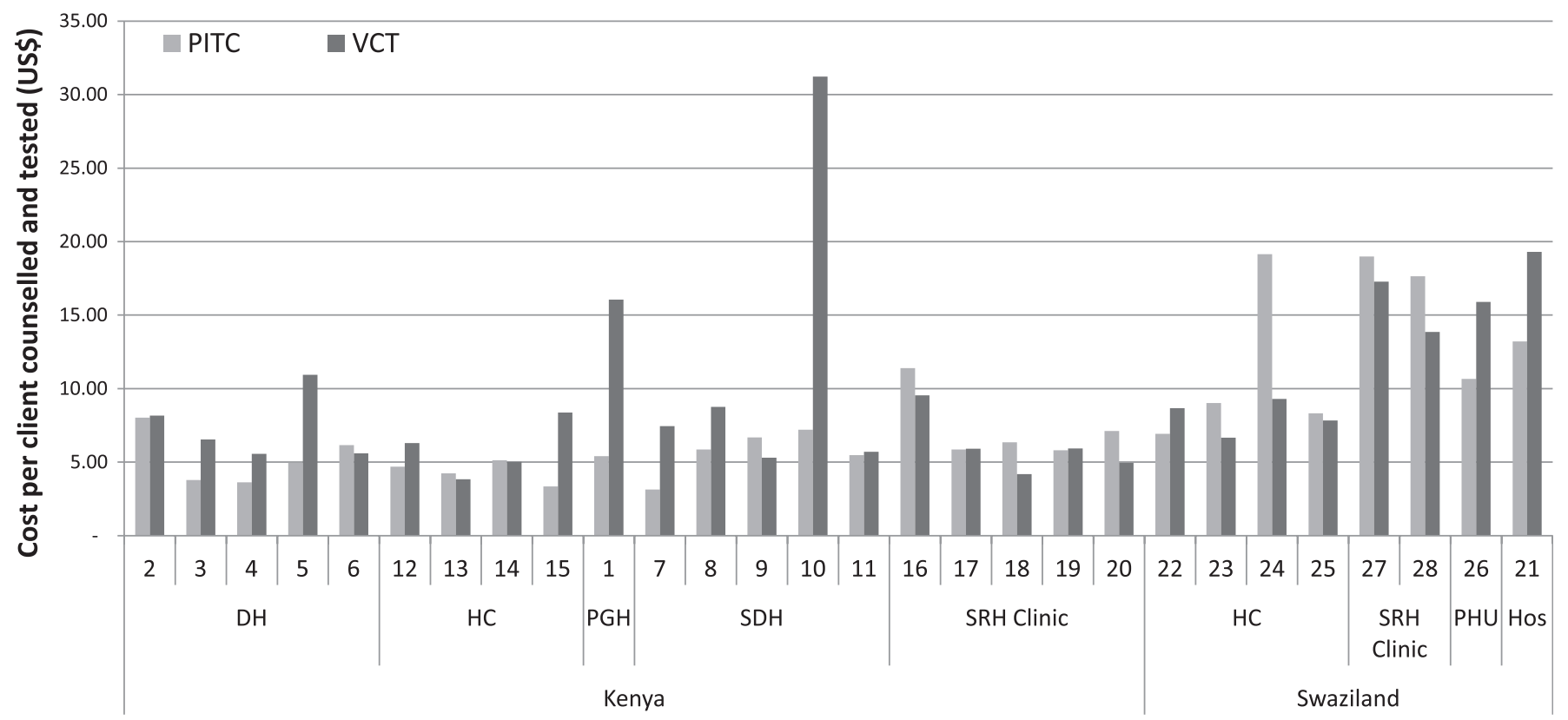

Figure 1 Variation in cost per client counselled and tested in Kenya and Swaziland (US\$ rates in 2009). DH, District hospital; HC, Health centre; PGH, Provincial general hospital; SDH, Sub-district hospital; PHU, Public health unit; Hos, Hospital. 
confirm in either setting whether positive tests were the first or a repeat result and, thus, draw conclusions about cost-effectiveness in terms of identifying new cases of HIV.

Our cost results are consistent with findings from other studies. A recent review of the efficiency gains of integrating HIV services into general health services identified a number of studies suggesting that the cost of integrated HCT in general health services is lower than that in VCT centres. ${ }^{17}$ However, all these were of a considerably smaller size than this study (1-4 sites), ${ }^{18-20}$ so no firm conclusion was possible.

Overall, the variation in unit costs between sites of a similar level suggests that there is considerable room for efficiency gain in HCT services. A substantial element of cost variation is accounted for by differences in personnel costs across settings. While there were some salary differentials between sites within each country, these were relatively minor, so the main driver of personnel costs was staff workload. Differences in staff workload can be partly explained by differences in model (such as group counselling) and, in part, by the organisation of service delivery, where staff are used for multiple purposes, and thus, are busier. However, it should be noted that in several of the facilities, the low costs of PITC are, in part, achieved by some staff facing exceptionally high workloads and, therefore, the 'efficiency' observed may come at a price of decreasing staff morale, possibly leading to services of poorer quality.

Not all VCTs, however, had a higher cost than PITC at the same site. The fact that some VCTs achieve a high workload demonstrates that there is nothing inherent in the VCT model that makes it less efficient in this regard. However, where VCT workloads are low, a stand-alone organisational structure, and limited training of counsellors may inhibit the efficient use of staff as they cannot be shared with other services. For this reason, more attention needs to be given to either better location or promoting VCT services in order that they are fully utilised; or to adding more services to VCT sites, to ensure that staff are used to a maximum to meet the needs of their clients. Recent efforts to include both TB-intensified case finding and the provision of FP through VCTs, ${ }^{21-23}$ thus offer feasible ways to improve the efficiency of these services.

A simple reading of our results would suggest that policy makers wishing to maximise the number of HIV-positive clients identified under budget constraints, should prioritise PITC. However, this would be incorrect, as our results also suggest the factors that drive efficiency (the demand for services, HIV prevalence and staffing) are context specific. Moreover, PITC and VCT services are not perfect substitutes for one another in all settings. Clientele attending VCT may be different from those accessing PITC. In particular, while integrated HCT within maternal and child health units is largely targeted at women of reproductive age, VCT services may be more able to meet the needs of other segments of the population, including adult men and adolescents. VCT may also be a better venue for activities, such as couple counselling, and may also be preferred by some people for other reasons, such as providing more specialised HIV services, and being better able to connect clients who test positive for HIV to services and peer-support groups. This suggests that while there is room for substantial improvement in efficiency in HCT services through the shared and integrated use of resources, this needs to be balanced with other programme objectives.

Finally, this study has a number of limitations that should be taken into account when interpreting our findings. First, the unit cost analysis was conducted retrospectively, which meant that staff observation could only be done at the end of the period for which we had full financial data. Second, we used

\section{Key messages}

PITC compares favourably with VCT in terms of cost per client C\&T and cost per client diagnosed as HIV positive in Kenya and Swaziland.

- Overall, the variation in unit costs between sites of similar levels suggests that there is considerable room for efficiency gain in HCT services.

- Considerations of how to deliver services efficiently need to be informed by local contextual factors, including HIV prevalence, service demand and human resource availability.

- Quality of HCT services should not be compromised either by overstretching providers or by undermining important considerations regarding consent, confidentiality and client rights.

routine monitoring data on service statistics to estimate unit costs. Variations in the completeness of monitoring data compiled may, therefore, have contributed to the variation in unit costs documented. Third, our sample only included 28 health facilities in Kenya and Swaziland. While this is much larger than previous studies, it remains insufficient for a full econometric analysis to quantitatively assess the influence of the different cost drivers. However, a second round of costing is currently underway to help further investigate cost drivers by examining changes over time. Fourth, our analysis does not include comparisons with other forms of HCT, such as homebased HCT. Lastly, the study did not measure the client costs incurred in accessing HCT services. The exclusion of these costs underestimates the true health systems and societal costs of accessing these services.

\section{CONCLUSIONS}

In the context of significant policy interest in optimising scarce HIV resources, this study demonstrates that there may be potential for substantial gains in efficiency in the provision of HCT services in both Kenya and Swaziland. The results can be used by those planning and providing both SRH and HIV services to improve programme efficiency and performance.

Our research highlights the importance of a programme science approach where planners and implementers jointly develop an intervention, and focus on operational research to strengthen the evidence base on how to improve the use of resources for HIV services. This model also provides important opportunities for the academic and international HIV community to systematically learn from large-scale HIV programme investments about how to efficiently deliver HIV programmes at scale.

Acknowledgements Our sincere gratitude to the Ministry of Health, Kenya, the Ministry of Health, Swaziland, and all the health facilities that participated in the study

Contributors CDO and AV collaborated in the writing of the first draft of the manuscript. CDO was also involved in all aspects of study design, data collection, analysis and interpretation of data for this study. AV also contributed to the study design, chose the main directions for data analysis, and participated in the interpretation of results. CM contributed to the study design, and was also involved in the data collection. All authors provided editorial input, and contributed to subsequent drafts of the manuscript and reviewed the final version prior to submission.

Funding This work was supported by the Bill and Melinda Gates Foundation, grant number 48733.

Correction notice This article has been corrected since it was published Online First. The sentence 'In a twice-daily bid to encourage HCT uptake to population groups with 
limited access to existing services, the Ministries of Health of both Kenya and Swaziland mandated PITC throughout the health sector in 2008.' has been updated to read 'In a bid to encourage HCT uptake to population groups with limited access to existing services, the Ministries of Health of both Kenya and Swaziland mandated PITC throughout the health sector in 2008.' Also, the repeat of the following sentence has been deleted "Financial costs represent actual expenditures on goods and services purchased, while economic costs include the estimated value of all resources, including donated or subsidised goods and services."

Competing interests None.

Ethics approval Ethics approval was provided by London School of Hygiene and Tropical Medicine; Population Council Institutional Review Board; Kenya Medical Research Institute National Ethical Review Committee; Swaziland Scientific Review Board.

Provenance and peer review Not commissioned; externally peer reviewed.

\section{REFERENCES}

1. UNAIDS. UNAIDS Report on the Global AIDS Epidemic. Geneva: UNAIDS, 2010.

2. De Cock KM, Brunnel R, Mermin J. Unfinished business-expanding HIV testing in developing countries. N Engl J Med 2006;354:440-2.

3. UNAIDS, World Health Organisation. UNAIDS/WHO Policy Statement on HIV Testing. Geneva: UNAIDS, 2004. http://www.who.int/rpc/research_ethics/ hivtestingpolicy en pdf.pdf (accessed 4 Jan 2011).

4. Bwambale $\mathbf{F M},{ }^{-}$ssali SN, Byaruhanga S, et al. Voluntary HIV counselling and testing among men in rural western Uganda: Implications for HIV prevention. BMC Public Health 2008:8:263.

5. Helleringer S, Kohler HP, Frimpong JA, et al. Increasing uptake of HIV testing and counselling among the poorest in sub-Saharan countries through home-based service provision. J Acquir Immune Defic Syndr 2009:51:185-93.

6. Kalichman SC, Simbayi LC. HIV testing attitudes, AIDS stigma, and voluntary HIV counselling and testing in a black township in Cape Town, South Africa. Sex Transm Infect 2003:79:442-7.

7. Sweat M, Gregorich S, Sangiwa G, et al. Cost-effectiveness of voluntary HIV-1 counselling and testing in reducing sexual transmission of HIV-1 in Kenya and Tanzania. Lancet 2000:356:113-21.

8. Obermeyer CM, Osborn M. The utilization of testing and counselling for HIV a review of the social and behavioral evidence. Am J Public Health 2007:97:1762-74.
9. WHO, UNAIDS, UNICEF. Towards universal access: scaling up priority HIVIAIDS interventions in the health sector. Progress Report. Geneva: WHO, UNAIDS and UNICEF, 2007 (accessed 6 Oct 2011).

10. Blanchard JF, Aral SO. Programme Science: an initiative to improve the planning implementation and evaluation of HIV/sexually transmitted infection prevention programmes. Sex Transm Infect 2011:87:2-3.

11. National AIDS Control Council. Kenya AIDS Indicator Survey 2007. Final Report. Kenya: NACC, 2009

12. Central Statistical Office Swaziland. 2006-2007 Swaziland Demographic Health Survey. Swaziland: Government of Swaziland, 2007.

13. National AIDS Control Council. Kenya National AIDS Strategic Plan 2009/10 2012/13: Delivering on Universal Access to Services. Nairobi, Kenya: NACC, 2009.

14. Government of the Kingdom of Swaziland. Monitoring the declaration of commitment on HIVIAIDS (UNGASS) Swaziland country report. Swaziland: UNAIDS, 2010.

15. Drummond MF, Sculpher MJ, Torrance GW, et al. Methods for the Economic Evaluation of Health Care Programs. 2nd edn. New York: Oxford University Press, 2005.

16. Oanda.com. Currency Converter. http://www.oanda.com/

17. Sweeney S, Obure CD, Maier CB, et al. Costs and efficiency of integrating HIV/AIDS services with other health services: a systematic review of evidence and experience. Sex Transm Infect 2012;88:85-99.

18. Twahir A, Maggwa B, Askew I. Integration of STI and HIVIAIDS services with MCHFP services: A case study of the Mkomani Clinic Society in Mombasa, Kenya. Kenya: Population Council, 1996

19. Liambila W, Askew I, Mwangi J, et al. Feasibility and effectiveness of integrating provider-initiated testing and counselling within family planning services in Kenya. AIDS 2009:23:S115-21.

20. Menzies N, Abang B, Wanyenze R, et al. The costs and effectiveness of four HIV counselling and testing strategies in Uganda. AIDS 2009;23:395-401.

21. Reynolds H, Beaston-Blaakman A, Burke H, et al. Integrating Family Planning Services into Voluntary Counselling and Testing Centres in Kenya. 2006:1-42. http://www. fhi360.org//NR/rdonlyres/evdwqnrtajii2jwyzdqom6xonyiurackisa57r3hakj4es4droeu fsknbpfitvplbc4wyi2jh3wmsh/FPVCTintegKenyaOR1.pdf (accessed 24 Jan 2012)

22. Gillespie D, Bradley $\mathrm{H}$, Woldegiorgis $\mathrm{M}$, et al. Integrating family planning into Ethiopian Voluntary testing and counselling programmes. Bull World Health Organ 2009;87:866-70.

23. Terris-Prestholt F, Kumaranayake L, Ginwalla R, et al. Integrating tuberculosis and HIV services for people living with HIV: costs of the Zambian ProTEST Initiative. Cost Eff Resour Alloc 2008;6:2. 